\title{
Cosmic-ray transport from AMS-02 B/C data: benchmark models and interpretation
}

\author{
Yoann Genolini*, M. Boudaud, P.-I. Batista, S. Caroff, L. Derome, J. Lavalle, A. \\ Marcowith, D. Maurin, V. Poireau, V. Poulin, S. Rosier, P. Salati, P. D. Serpico, M. \\ Vecchi \\ Service de Physique Théorique, Université Libre de Bruxelles, Boulevard du Triomphe, CP225, \\ 1050 Brussels, Belgium \\ E-mail: yoann.genolini@ulb.ac.be
}

\begin{abstract}
We present new benchmark scenarios for Galactic cosmic-ray propagation in the GV-TV rigidity range, based on fits to the AMS-02 B/C data with the USINE V3.5 propagation code. We employ a new fitting procedure, cautiously taking into account data systematic error correlations in different rigidity bins and considering Solar modulation potential and leading nuclear cross section as nuisance parameters. We delineate specific low, intermediate, and high-rigidity ranges that can be related to both features in the data and peculiar microphysics mechanisms resulting in spectral breaks. We single out a scenario which yields excellent fits to the data and includes all the presumably relevant complexity, the BIG model. This model has two limiting regimes: (i) the SLIM model, a minimal diffusion-only setup, and (ii) the QUAINT model, a convectionreacceleration model where transport is tuned by non-relativistic effects. All models lead to robust predictions in the high-energy regime $(\gtrsim 10 \mathrm{GV})$, i.e. independent of the propagation scenario: at $1 \sigma$, the diffusion slope $\delta$ is $[0.43-0.53]$, whereas $K_{10}$, the diffusion coefficient at $10 \mathrm{GV}$, is $[0.26-0.36] \mathrm{kpc}^{2} \mathrm{Myr}^{-1}$; we confirm the robustness of the high-energy break, with a typical value $\Delta_{h} \sim 0.2$. We also find a hint for a similar (reversed) feature at low rigidity around the $\mathrm{B} / \mathrm{C}$ peak $(\sim 4 \mathrm{GV})$ which might be related to some effective damping scale in the magnetic turbulence. Initialization files used for the analysis, along with the resulting best-fit values and covariance matrix of best-fit parameters are provided with USINE V3.5.
\end{abstract}

36th International Cosmic Ray Conference -ICRC2019-

July 24th - August 1st, 2019

Madison, WI, U.S.A.

${ }^{*}$ Speaker. 


\section{Introduction}

The last decade in direct cosmic-ray (CR) detection experiments has been characterized by a major improvement in the precision of the data available, and by an extension of the covered dynamical range. In particular, with the AMS-02 data the community has to deal for the first time with percent level precision and a welcomed redundancy in the measurements. As long established, a flux ratio of elements present but in traces in the solar system material and interstellar medium (ISM), such as Lithium, Beryllium, Boron ("secondaries"), to abundant species like Carbon or Oxygen ("primaries") is extremely sensitive to propagation parameters.

In this proceeding, we focus on the AMS-02 B/C data [1] and investigate how much they can constrain CR transport, aiming at defining new benchmark models. An important and relatively new issue of such analysis is that data systematic errors are often dominant over statistical ones. This requires a change of perspective in well-established practices of analyzing the data, beginning with a relatively simple and homogeneous data sample. In [2], we performed an analysis of the high-rigidity range of the AMS-02 B/C ratio [1], finding evidence for a diffusive origin of the observed spectral break, at the same rigidity scale inferred from a similar feature in the proton and helium CR fluxes [3], i.e. $\sim 300 \mathrm{GV}$.

Here, we move several steps beyond our previous analysis [2], presenting a complete analysis aiming at constraining CR propagation and at proposing new benchmark setups. First, we rely on an improved analysis of the B/C data by the AMS-02 collaboration [4]. We further benefit from additional data on the primary species to constrain the break independently from the $\mathrm{B} / \mathrm{C}$ ratio- using the $\mathrm{C}$ and $\mathrm{O}$ fluxes [5] which are most contributing species to $\mathrm{B}$ production [6], but were not available to Ref. [2]. Second, we follow the new methodology proposed in [7] to analyze the AMS-02 data, carefully accounting for partial correlations in systematic errors and theoretical uncertainties. Third, we propose a new generic propagation model (dubbed BIG in the following), with a number of parameters that should be sufficient to describe all key features currently present in the data. In addition to a high-rigidity break, a modification of the diffusion coefficient at low rigidity is enabled ( $\lesssim 5 \mathrm{GV}$ ), with two limiting cases (dubbed SLIM and QUAINT): this allows us to assess the relative discriminating strength of the data in this energy range and to shed new light on propagation in the low-rigidity regime, where a second diffusion break might be present.

\section{Cosmic-ray transport}

CR propagation is based on the generic propagation equation (Eq.(1) of [8]) that we further solve semi-analytically in the framework of the USINE code [9]. More precisely, we assume a 1D propagation model ${ }^{1}$, where the magnetic halo confining the CRs is an infinite slab in the radial direction and of half-height $L$. The sources of CRs and the ISM gas which they scatter off are taken homogeneous in an infinitely thin disk at $z=0$, with an effective half-height $h=100 \mathrm{pc}$. Energy losses are also considered to be localized in the disk too. For the nuclear production and spallation cross sections, we use as reference the sets of tables from the Galprop package. Finally, switching from the interstellar (IS) CR flux predictions to the top-of-atmosphere (TOA) ones is achieved by means of the force-field approximation [10,11].

\footnotetext{
${ }^{1}$ In appendix C of Ref.[8], we deal with a 2D model.
} 
An important physical ingredient to all propagation models is the diffusion coefficient, which describes the scattering of CRs off magnetic turbulence. We assume that it can be taken as a scalar function, homogeneous and isotropic all over the magnetic slab. On the theory side, it is expected that the magnetic turbulence responsible for CR diffusion has different scaling behaviors in $k$-space, as a consequence of various possible phenomena. For instance, the turbulence power spectrum can be dominated by different sources depending on the dynamical range, with the resulting "twozone" models known to provide good fits to the data $[12,13]$. A very appealing scenario proposed in Refs. $[14,15]$ relies on the streaming instability. At rigidities beyond a few hundreds of GV, CRs diffuse on the turbulence injected on large scales by supernova bubbles, whereas at lower rigidity this turbulence cascades down until crossing the rigidity scale where the turbulence induced by CRs themselves takes over. This naturally generates a break like the one observed in the CR spectra and discussed just above. On the other hand, it is known that the CR spectra observed at low rigidity by the Voyager I spacecraft [16] have a spectral slope rather different from the slope at intermediate rigidities. Due to the CR-wave coupling, any phenomenon with a low-rigidity characteristic scale, affecting either propagation or injection, may thus be at the origin of correlated changes in the CR spectra and the diffusion coefficient.

We want to capture the possibility that the diffusion coefficient departs from a single power law, which is justified by both theoretical arguments and observational evidence. Thus we introduce the following phenomenological form for the diffusion coefficient:

$$
K(R)=\underbrace{\beta^{\eta}}_{\text {non-relativistic regime }} K_{10} \underbrace{\left\{1+\left(\frac{R}{R_{\mathrm{l}}}\right)^{\frac{\delta_{1}-\delta}{s_{\mathrm{l}}}}\right\}^{s_{1}}}_{\text {low-rigidity regime }} \underbrace{\left\{\frac{R}{\left(R_{10} \equiv 10 \mathrm{GV}\right)}\right\}^{\delta}}_{\text {intermediate regime }} \underbrace{\left\{1+\left(\frac{R}{R_{\mathrm{h}}}\right)^{\frac{\delta-\delta_{\mathrm{h}}}{\mathrm{s}_{\mathrm{h}}}}\right\}^{-s_{\mathrm{h}}}}_{\text {high-rigidity regime }}
$$

In the above equation, $\beta=v / c$ is the dimensionless CR speed, and $R_{1 / \mathrm{h}}$ is the location of the low/high-rigidity break, while $R_{10}$ is an intermediate rigidity (here taken at $10 \mathrm{GV}$ on purpose) such that $R_{1}<R_{10}<R_{\mathrm{h}}\left(R_{1} \ll R_{\mathrm{h}}\right)$. We then get the scaling $K(R) \propto \beta^{\eta} R^{\delta_{1}}$ in the limit $R \ll R_{\mathrm{l}}$, and the scaling $K(R) \propto R^{\delta_{\mathrm{h}}}$ in the limit $R \gg R_{\mathrm{h}}$. Therefore, $\delta_{\mathrm{l}}, \delta$, and $\delta_{\mathrm{h}}$ simply describe the diffusion spectral indices in the low-, intermediate-, and high-rigidity regime, respectively. The parameter $s_{1}$ $\left(s_{\mathrm{h}}\right)$ characterizes how fast the spectral change proceeds around $R_{\mathrm{l}}\left(R_{\mathrm{h}}\right)$.

\section{Benchmark models}

In the most general case, the free parameters featuring the propagation modeling that we have introduced above are the following: $L$ for the magnetic halo size; $K_{10}, \delta, \eta, R_{1}, \delta_{1}$ (equivalently $\Delta_{\mathrm{l}}$ ), $s_{1}, R_{\mathrm{h}}, \Delta_{\mathrm{h}}$ (equivalently $\delta_{\mathrm{h}}$ ), and $s_{\mathrm{h}}$ for the diffusion coefficient; $V_{\mathrm{A}}$ for reacceleration; $V_{\mathrm{c}}$ for convection. This is a 12-parameter space, hence a huge configuration volume to explore.

Based on previous studies, we can further fix $L$ which is highly correlated with $K_{10}{ }^{2}$. We set $L$ to $10 \mathrm{kpc}$ in the following. Moreover, we will see that the determination of the three parameters describing the high-rigidity break benefits from fits including primary species, see Sect. 4. Finally,

\footnotetext{
${ }^{2}$ Note that in [8], we provide a detailed discussion of the dependence with $L$ in Appendix C and D.
} 
without loss of generality, we fix the smoothing low-rigidity break parameter $s_{1}=0.05$, which amounts to consider a fast transition. This is however not critical to the fit. Hence, we are left with 7 free parameters. From these 7 parameters, we design three different benchmark propagation models which may be related to quite different limiting regimes of the underlying low-rigidity microphysics:

- The BIG model: includes the whole setup introduced above which allows us to probe the low-rigidity processes with the largest flexibility and complexity. This model has a total of 6 parameters in the minimal configuration $\left(\eta=1 \mathrm{fixed}^{3}\right): K_{10}, \delta, \delta_{1}, R_{1}, V_{\mathrm{A}}, V_{\mathrm{c}}$.

- The SLIM model: a subpart of BIG, which discards convection and reacceleration as major players at low rigidity $\left(V_{\mathrm{A}}=V_{\mathrm{c}}=0 \mathrm{~km} / \mathrm{s}\right)$. Its advantage is that it comes with a fully analytical solution to the transport equation. It has 4 free parameters: $K_{10}, \delta, \delta_{1}, R_{1}$.

- The QUAINT model: a subpart of the BIG model too, which aims at describing the lowrigidity features mostly in terms of reacceleration and convection. This model is inspired by the min-med-max models $[17,18]$, with a large $V_{\mathrm{A}}$, in combination with a non-trivial value of $\eta \lesssim 0$ needed to provide decent fits to the data ${ }^{4}$. It has 5 free parameters: $K_{10}, \delta, \eta, V_{\mathrm{A}}$, $V_{\mathrm{c}}$.

\section{Fitting strategy}

Fits of the benchmark propagation models (BIG, SLIM, and QUAINT) are performed with the MINUIT package [19] interfaced with the USINE code [9], and in particular, asymmetric error bars on the parameters rely on the MINOS algorithm. For more technical details and subtleties on the setup and the analysis, we refer the reader to Ref. [7].

Modeling uncertainties: We assume the primary injection to follow a universal power law in rigidity with index $\alpha$. The secondary component is computed by a full spallation network using the Galprop cross-section parameterization which provides the best agreement with the data. The uncertainties on spallation cross sections are satisfactorily taken into account using only the ${ }^{12} \mathrm{C}+\mathrm{H} \rightarrow{ }^{11} \mathrm{~B}$ production cross section as nuisance parameter with the "normalization, slope and shape" (NSS) strategy (see [7]). The solar modulation of CRs is described in the force-field approximation, for which the Fisk potential $\phi_{\mathrm{F}}$ is averaged over the AMS-02 B/C data taking period. Based on [20], we set $\phi_{\mathrm{F}}$ as a nuisance parameter of mean value $730 \mathrm{GV}$ and dispersion $\sigma_{\phi_{\mathrm{F}}}=100 \mathrm{MV}$.

Data errors: Contrary to the commonly used total errors (obtained by summing systematics and statistics in quadrature), here we reconstructed a parametric form of the covariance matrix from the information provided in the Supplemental Material of the AMS-02 B/C analysis [4]. This is a major improvement since the AMS-02 data are mostly dominated by systematics (below $\sim 100$ GV).

\footnotetext{
${ }^{3}$ The freedom allowed by $\eta$ is actually degenerated with the one of the low-rigidity break.

${ }^{4}$ if we had fixed $\eta=1$ in the QUAINT model, its fit quality would degrade, and it would be rejected at $>2 \sigma$ with respect to the BIG and SLIM models in the following.
} 
$C$ and $O$ primaries: It has been noted already that recent data show an indication for a highrigidity break in the diffusion coefficient. However, these data are at present still far for providing us with the precise characteristics of this feature. Since we want to provide reference values for the parameters controlling the low- and intermediate-rigidity regimes, it is a natural choice to use the high-rigidity break parameters as nuisance parameters. To establish the plausible range over which to vary them, we use the AMS-02 C and O fluxes because: (i) They are by far the main progenitors of the $\mathrm{B}$ and $\mathrm{C}$ fluxes entering the $\mathrm{B} / \mathrm{C}$ ratio, (ii) Fitting them allows us to determine a plausible value of the common spectral index of nuclei $\alpha$ as well as to check their consistency with the parameters obtained with the $\mathrm{B} / \mathrm{C}$ analysis. The range found is actually more constraining that from the $\mathrm{B} / \mathrm{C}$ alone since the $\mathrm{B}$ flux, which dominates the $\mathrm{B} / \mathrm{C}$ statistical error at high rigidity, is more than one order of magnitude scarcer than the $\mathrm{C}$ and $\mathrm{O}$ ones.

The fitting procedure: The technical implementation of the fits proceeds by iteration. After fixing the (low- and intermediate-rigidity) propagation parameters with a first fit of the $\mathrm{B} / \mathrm{C}$ ratio (as described above, i.e. with $\phi_{\mathrm{F}}$ and the ${ }^{12} \mathrm{C}+\mathrm{H} \rightarrow{ }^{11} \mathrm{~B}$ production cross section taken as nuisance parameters), we perform a combined fit of the AMS-02 $\mathrm{C}$ and $\mathrm{O}$ fluxes keeping the following parameters as free parameters: source-term normalizations, power-law dependence in rigidity $\alpha$, and break parameters $\left(R_{\mathrm{h}}, s_{\mathrm{h}}\right.$, and $\left.\Delta_{\mathrm{h}}\right)$. We then use the best-fit values of the break parameters and associated covariance matrix as nuisance parameters in a new B/C fit, keeping also $\alpha$ fixed to its best-fit value. In practice, only a couple of iterations are needed to get the parameters compatible between two consecutive iterations. The results discussed below are the outcome of this procedure.

\section{Results}

The best-fit values and errors on the three model parameters (BIG, SLIM, and QUAINT) are reported in Tab. 1. In the first block, we report the diffusion parameters $\delta$ and $K_{10}$ common to all models, which control the intermediate-rigidity regime. We then report the low-rigidity parameters, which are different (both in nature and number) between QUAINT, on one side, and, BIG and SLIM, on the other. The high-rigidity break parameters, fixed following the nuisance procedure, are reported at the bottom of the Table. In all these fits, nuisance parameters vary within reasonable pre-assigned intervals. Our best-fit curves are reported in Fig. 1 for the three models. Note that all models lead to analogous curves and fit quality.

The similar fit quality of the BIG and SLIM models indicates that the additional free parameters present in the former are actually unnecessary to describe the data: if the fit allows for a low-rigidity break, there is but a minor and currently unnecessary role played by $V_{\mathrm{c}}$ and $V_{\mathrm{A}}$. We note that, compared to SLIM, the benchmark BIG and QUAINT have respectively a weaker and no break at low rigidity, although the latter is partly mimicked by the spectral distortions of the cross section in nuisance and a value of $\eta<1$ in QUAINT. This tends to provide additional support to the possible presence of a low-rigidity break in the diffusion coefficient.

Also, it is important to notice that the parameters common to the three models are found with values compatible within $\sim 1 \sigma$. This suggests that the diffusive properties at intermediate rigidities are constrained rather robustly by the data, independently of the specific scenario within which the low-rigidity behavior is interpreted and fitted. Note that the value found for $\delta$ appears 


\begin{tabular}{lccc}
\hline \hline Parameters & BIG & SLIM & QUAINT \\
\hline \hline$\chi^{2} /$ dof & $61.7 / 61=1.01$ & $61.8 / 63=0.98$ & $62.1 / 62=1.00$ \\
\hline \multicolumn{4}{c}{ Intermediate-rigidity parameters } \\
$K_{10}\left[\mathrm{kpc}^{2} \mathrm{Myr}^{-1}\right]$ & $0.30_{-0.04}^{+0.03}$ & $0.28_{-0.02}^{+0.02}$ & $0.33_{-0.06}^{+0.03}$ \\
$\delta$ & $0.48_{-0.03}^{+0.04}$ & $0.51_{-0.02}^{+0.02}$ & $0.45_{-0.02}^{+0.05}$ \\
\hline \multicolumn{4}{c}{ Low-rigidity } \\
$V_{\mathrm{c}}\left[\mathrm{km} \mathrm{s}^{-1}\right]$ & $0^{+7.4}$ & N/A & $0.0^{+8}$ \\
$V_{\mathrm{A}}\left[\mathrm{km} \mathrm{s}^{-1}\right]$ & $67_{-67}^{+24}$ & N/A & $101_{-15}^{+14}$ \\
$\eta$ & 1 (fixed) & 1 (fixed) & $-0.09_{-0.57}^{+0.35}$ \\
$\delta_{\mathrm{l}}$ & $-0.69_{-1.26}^{+0.61}$ & $-0.87_{-0.31}^{+0.33}$ & N/A \\
$R_{\mathrm{l}}[\mathrm{GV}]$ & $3.4_{-0.9}^{+1.1}$ & $4.4_{-0.2}^{+0.2}$ & N/A \\
\hline \hline \multicolumn{5}{c}{ High-rigidity break parameters } \\
$\Delta_{\mathrm{h}}$ & (nuisance parameters) \\
$R_{\mathrm{h}}[\mathrm{GV}]$ & 0.18 & 0.19 & \\
$s_{\mathrm{h}}$ & 247 & 237 & 0.17 \\
\hline
\end{tabular}

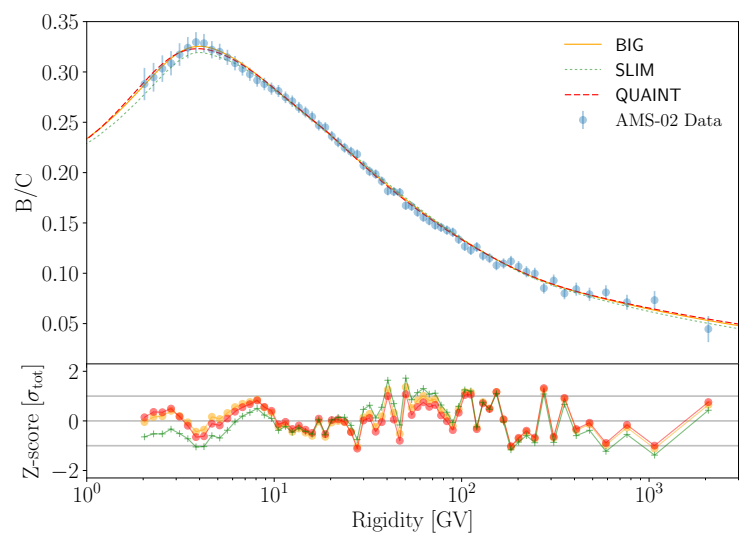

Table 1: Best-fit parameter values and uncertainties for the three Figure 1: Best fit B/C curve for models BIG, SLIM, and benchmark models BIG, SLIM, and QUAINT and corresponding QUAINT. Results for the best fit parameter values are given in $\chi^{2} /$ dof. The high-rigidity break parameters are nuisance parame- Tab. 1 . The bottom panel shows the $Z$-score, only relevant for visual ters in the fit, and their preferred post-fit values are also quoted for inspection, since the $\chi^{2}$ is computed accounting for correlations, usthe sake of completeness. ing the covariance matrix of the data.

closer to a Kraichnan turbulence spectrum $(\delta \simeq 0.5)$ than to a Kolmogorov one ( $\delta \simeq 1 / 3)$, although this conclusion should not be overstated since the model involves an effective isotropic diffusion coefficient.

Concerning the low-rigidity regime, however, there are several important caveats, which suggest some prudence to avoid over-interpreting the values found. First of all, while there is a clear indication for a different regime of propagation at low rigidity, the "hardest" parameters to interpret ( $\eta$ and $\delta_{1}$ ) are actually heavily influenced by the one or two lowest-rigidity points (see Appendix B of [8]). There is simply not enough of a baseline at low rigidity in the AMS-02 data to unambiguously measure the slope in this range. Another point to keep in mind is that the low-rigidity range is quite influenced by the uncertainties in the nuclear cross sections. Indeed, including the nuisance parameters for the production cross section increases the $1 \sigma$ uncertainties on $\eta$ (QUAINT) by $50 \%$, and on $R_{l}$ and $\delta_{l}(\mathrm{BIG})$ by $90 \%$. The only model-independent conclusion that we can safely make on the low-rigidity range is that multiple models can account for the observations, with rather different physical interpretations possible.

\section{Conclusions and perspectives}

In this study, we have defined benchmark models and ranges of parameters with a careful statistical analysis of the AMS-02 B/C data. We have checked different theoretical frameworks differing in the treatment of transport at low rigidities with a major (model QUAINT) or a negligible (model SLIM) role played by reacceleration. Both models are limiting cases of a more general model (BIG). We have made sure that issues like numerical stability, the effects of cross sections uncertainties, the bin-to-bin correlation of systematic errors are handled sufficiently well not to bias significantly the conclusions.

For the time being, either model can describe with comparable performances the low-rigidity regime. The degeneracies with nuisance parameters impact both the best fits and uncertainties, in particular the ones controlling the energy shape of cross sections. Obviously, any discrimination 
between the two sets of models must be based on complementary data or arguments, such as the (astro)physical plausibility of the parameters found. However, finding a break in the diffusion coefficient at low rigidity should not come so much as a surprise, since this feature, possibly related to some damping in the turbulence spectrum and the subsequent increase of the CR mean free path, is expected from theoretical grounds. In this respect, a careful study of low energy data complimentary to AMS-02 ones (e.g. from ACE-CRIS and Voyager I), could certainly help in drawing more robust conclusions on the properties and the nature of this break. Besides extracting reference propagation parameters we have also confirmed and strengthened our conclusions in [2], that the high-rigidity data can be consistently interpreted as a consequence of a break in the diffusive coefficient, in agreement with AMS-02 high-energy primaries spectra.

Following this study, the most pressing issue is of course to test the reference models provided here against other secondary data (e.g., Li, Be, antiprotons, positrons). In particular, we have recently show in [21] that antiprotons are consistent with a secondary astrophysical origin.

\section{References}

[1] M. Aguilar et al. Precision Measurement of the Boron to Carbon Flux Ratio in Cosmic Rays from 1.9 GV to 2.6 TV with the Alpha Magnetic Spectrometer on the International Space Station. Phys. Rev. Lett., 117(23):231102, December 2016.

[2] Y. Génolini, P. D. Serpico, M. Boudaud, S. Caroff, V. Poulin, L. Derome, J. Lavalle, D. Maurin, V. Poireau, S. Rosier, P. Salati, and M. Vecchi. Indications for a high-rigidity break in the cosmic-ray diffusion coefficient. Phys. Rev. Lett., 119(24):241101, December 2017.

[3] M. Aguilar et al. Precision Measurement of the Proton Flux in Primary Cosmic Rays from Rigidity 1 GV to 1.8 TV with the Alpha Magnetic Spectrometer on the International Space Station. Phys. Rev. Lett., 114(17):171103, May 2015.

[4] M. Aguilar et al. Observation of new properties of secondary cosmic rays lithium, beryllium, and boron by the alpha magnetic spectrometer on the international space station. Phys. Rev. Lett., 120(2):021101, January 2018.

[5] M. Aguilar et al. Observation of the identical rigidity dependence of he, $c$, and o cosmic rays at high rigidities by the alpha magnetic spectrometer on the international space station. Phys. Rev. Lett., 119:251101, Dec 2017.

[6] Y. Génolini, D. Maurin, I. V. Moskalenko, and M. Unger. Current status and desired precision of the isotopic production cross sections relevant to astrophysics of cosmic rays: $\mathrm{Li}, \mathrm{Be}, \mathrm{B}, \mathrm{C}$, and $\mathrm{N}$. Phys. Rev. C, 98(3):034611, September 2018.

[7] L. Derome, D. Maurin, P. Salati, M. Boudaud, Y. Génolini, and P. Kunzé. Fitting b/c cosmic-ray data in the ams-02 era: a cookbook. arXiv e-prints, April 2019.

[8] Y. Génolini et al. Cosmic-ray transport from AMS-02 boron to carbon ratio data: Benchmark models and interpretation. Phys. Rev., D99(12):123028, 2019.

[9] D. Maurin. Usine: semi-analytical models for galactic cosmic-ray propagation. ArXiv e-prints, July 2018.

[10] L. J. Gleeson and W. I. Axford. Solar Modulation of Galactic Cosmic Rays. ApJ, 154:1011, December 1968. 
[11] L. A. Fisk. Solar modulation of galactic cosmic rays, 2. J. Geophys. Res., 76:221, 1971.

[12] N. Tomassetti. Origin of the Cosmic-Ray Spectral Hardening. ApJ, 752:L13, June 2012.

[13] Y.-Q. Guo and Q. Yuan. Understanding the spectral hardenings and radial distribution of galactic cosmic rays and fermi diffuse $\gamma$ rays with spatially-dependent propagation. Phys. Rev. D, 97(6):063008, March 2018.

[14] P. Blasi, E. Amato, and P. D. Serpico. Spectral Breaks as a Signature of Cosmic Ray Induced Turbulence in the Galaxy. Phys. Rev. Lett., 109(6):061101, August 2012.

[15] R. Aloisio, P. Blasi, and P. D. Serpico. Nonlinear cosmic ray galactic transport in the light of ams-02 and voyager data. $A \& A, 583:$ A95, November 2015.

[16] A. C. Cummings, E. C. Stone, B. C. Heikkila, N. Lal, W. R. Webber, G. Jóhannesson, I. V. Moskalenko, E. Orlando, and T. A. Porter. Galactic cosmic rays in the local interstellar medium: Voyager 1 observations and model results. ApJ, 831:18, November 2016.

[17] D. Maurin, F. Donato, R. Taillet, and P. Salati. Cosmic Rays below Z=30 in a Diffusion Model: New Constraints on Propagation Parameters. ApJ, 555:585-596, July 2001.

[18] F. Donato, N. Fornengo, D. Maurin, P. Salati, and R. Taillet. Antiprotons in cosmic rays from neutralino annihilation. Phys. Rev. D, 69(6):063501, March 2004.

[19] F. James and M. Roos. Minuit - a system for function minimization and analysis of the parameter errors and correlations. Computer Physics Communications, 10:343-367, December 1975.

[20] A. Ghelfi, F. Barao, L. Derome, and D. Maurin. Non-parametric determination of H and He interstellar fluxes from cosmic-ray data. A\&A, 591:A94, June 2016.

[21] M. Boudaud, Y. Génolini, L. Derome, J. Lavalle, D. Maurin, P. Salati, and P.D. Serpico. AMS-02 antiprotons are consistent with a secondary astrophysical origin. 2019. 